\title{
Logarithmically convex Reinhardt domains
}

LUDMILA BOURCHTEIN

ANDREI BOURCHTEIN

\begin{abstract}
The domains of certain types, such as Reinhardt ones, are important in different problems of theory of functions of several complex variables. For instance, any power series of several complex variables converges in the complete logarithmically convex Reinhardt domain. In this article we prove the logarithmic convexity of complete convex Reinhardt domains.
\end{abstract}




\section{Resumo}

Os domínios de certos tipos, tais como os de Reinhardt, são importantes em diferentes problemas de teoria de funções de várias variáveis complexas. Por exemplo, qualquer série de potências de várias variáveis complexas converge num domínio completo logaritmicamente convexo de Reinhardt. Neste artigo demonstramos que os domínios completos convexos de Reinhardt são logaritmicamente convexos.

Keywords: several complex variables, Reinhardt domain, logarithmic convexity

\section{Introductory definitions}

This work is continuation of the research presented in [1] regarding some properties of the Reinhardt and Hartogs domains used for global characterization of domains of holomorphy, which is one of the core problems in the modern theory of several complex variables [2, 3]. In particular, an investigation of convexity properties of Reinhardt domains allows to characterize the domains of convergence of the power series in the case of several complex variables. Here, we will apply the Reinhardt and logarithmic transformations to prove the logarithmic convexity of the convex Reinhardt domains.

Note that this result is well known (e.g. [3]), however the usual proof of this property is based on large preliminar theoretical material including investigation of the domains of convergence of a power series in $C^{n}$, an introduction of the concept of a domain of holomorphy and a study of their properties, a determination of connection between a domain of holomorphy and a domain of convergence of a power series. In this article the main result is proved by analyzing only geometrical properties of the considered domains.

First we introduce some pertinent definitions. Some definitions were given in [1], but we decided to recall these for completeness of exposition. 
Let us consider the complex $n$-dimensional linear space $C^{n}$ over the complex field $C$ with Euclidean norm introduced by formula

$$
|z|^{2}=\sum_{k=1}^{n}\left|z_{k}\right|^{2},
$$

where $z=\left(z_{1}, z_{2}, \ldots, z_{n}\right)=\left\{z_{k}\right\}_{k=1}^{n}$ is the element of the space $C^{n}$, $z_{k} \in C$ is the $k$-th coordinate and $\left|z_{k}\right|$ is its absolute value. This norm coincides with Euclidean norm of vector $z$ in the space $R^{2 n}$.

Definition. A Reinhardt domain with center $a \in C^{n}$ satisfies the following property: if point $z^{0}=\left\{z_{k}^{0}\right\}_{k=1}^{n}$ belongs to this domain then all points in the form

$$
z=\left\{a_{k}+\left(z_{k}^{0}-a_{k}\right) e^{i \theta_{k}}\right\}_{k=1}^{n}, \quad 0 \leq \theta_{k}<2 \pi,
$$

belong to this domain, too $[4,5]$.

Definition. A Reinhardt domain with center $a \in C^{n}$ is complete if together with any point $z^{0}$ it contains all points $z=\left\{z_{k}\right\}_{k=1}^{n}$ which satisfy inequalities

$$
\left|z_{k}-a_{k}\right| \leq\left|z_{k}^{0}-a_{k}\right|, \forall k=1, \ldots, n
$$

Otherwise, Reinhardt domain is incomplete $[4,5]$.

Without loss of generality we can consider the Reinhardt domains with center in the origin $(a=0)$. In this case, if a point $z=\left\{z_{k}\right\}_{k=1}^{n}$ belongs to a such domain, then the same happen to all points with the same modulus of components $\left|z_{k}\right|, k=1, \ldots, n$ and any argument. formula

Definition. A Reinhardt transformation is determined by 


$$
z \rightarrow \alpha(z)=\left(\left|z_{1}\right|,\left|z_{2}\right| \ldots,\left|z_{n}\right|\right)=\left\{\left|z_{k}\right|\right\}_{k=1}^{n}
$$

It maps complex $n$-dimensional space $C^{n}$ into real $n$-dimensional space $R^{n}$, more precisely onto $R_{+}^{n}=R_{+} \times \ldots \times R_{+}$, where $R_{+}=[0 ;+\infty)[5]$.

Reinhardt transformation $\alpha: C^{n} \rightarrow R_{+}^{n}$ maps a Reinhardt domain $D$ into set of points $D_{+} \subset R_{+}^{n}$ which is called the Reinhardt image (or diagram) of the domain $D$. If $D$ is a complete Reinhardt domain, then the set $D_{+}$contains together with any point $\left\{\left|z_{k}^{0}\right|\right\}_{k=1}^{n}$ entire rectangular parallelepiped $\left\{\left|z_{k}\right| \leq\left|z_{k}^{0}\right|, \forall k=1, \ldots, n\right\}$. This diagram characterizes completely the Reinhardt domains and makes possible its geometric representations in the cases $n=2$ and $n=3$.

Definition. Let

$$
z \rightarrow \lambda(z)=\left(\ln \left|z_{1}\right|, \ldots, \ln \left|z_{n}\right|\right)
$$

be logarithmic transformation of a set $\left\{z \in C^{n}: z_{1} \cdot z_{2} \cdot \ldots \cdot z_{n} \neq 0\right\}$ onto space $R^{n}$. Logarithmic image of set $M \subset C^{n}$ is a set $M^{*}=\lambda\left(M_{0}\right)$ where $M_{0}=\left\{z \in M: z_{1} \cdot \ldots \cdot z_{n} \neq 0\right\}[5]$.

Definition. A set $M$ is logarithmically convex if its logarithmic image $M^{*}$ is convex set in the space $R^{n}[3,5]$.

\section{Logarithmic convexity of Reinhardt domains}

A logarithmically convex set need not be a convex set. For example, a set of points $z=\left(z_{1}, z_{2}\right) \in C^{2}$ satisfying condition 
$\left|z_{1} z_{2}\right|<1$ is not convex (Fig.1 represents Reinhardt diagram of this set). At the same time logarithmic image of this set is a convex set (see Fig.2).

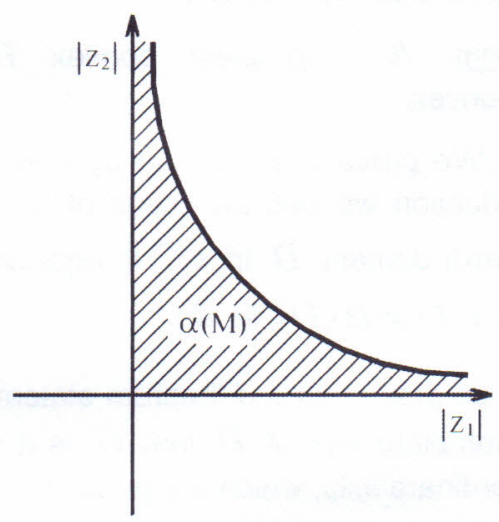

Fig. 1 - Reinhardt diagram of the set $\left\{z \in C^{2}:\left|z_{1} z_{2}\right|<1\right\}$

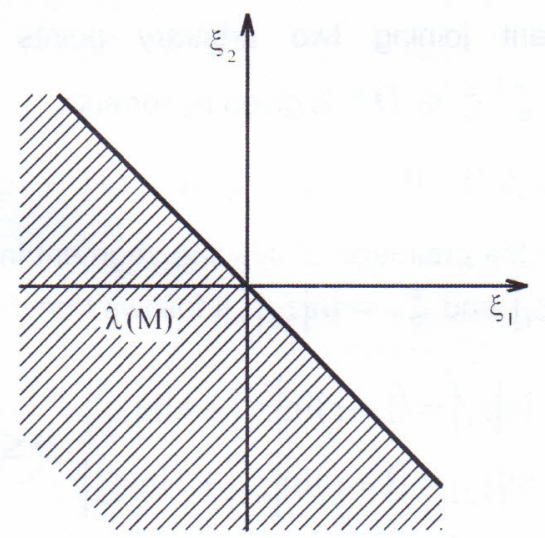

Fig. 2 - Logarithmic image of the set $\left\{z \in C^{2}:\left|z_{1} z_{2}\right|<1\right\}$

Ciência \& Natura, Santa Maria, 25: 7 - 22, 2003

11 
We will show that the inverse statement is true for complete Reinhardt domains. In what follows, we will use the following result demonstrated in [1]:

Lemma. A complete Reinhardt domain $D \subset C^{n}$ is convex in $C^{n}$ if, and only if, its image $G=\alpha(D)$ under Reinhardt transformation (1) is a convex set in $R^{n}$.

Theorem. Any complete convex Reinhardt domain is logarithmically convex.

Proof. We prove this theorem by mathematical induction. At each step of induction we use the result of Lemma that convexity of complete Reinhardt domain $D$ in $C^{n}$ is equivalent to convexity of its Reinhardt diagram $G=\alpha(D)$ in $R^{n}$.

1. Let us prove that the theorem statement is true for $n=2$. It follows from completeness of $D$ that $G$ is a set containing the line segments of coordinate axis, which are parts of its boundary.

We denote by $D^{*}$ a logarithmic image of domain $D$ under transformation (2) for $n=2$

$$
z \rightarrow \lambda(z)=\left(\ln \left|z_{1}\right|, \ln \left|z_{2}\right|\right)=\left(\xi_{1}, \xi_{2}\right)
$$

A line segment joining two arbitrary points $\xi^{1}=\left(\xi_{1}^{1}, \xi_{2}^{1}\right)$ and $\xi^{2}=\left(\xi_{1}^{2}, \xi_{2}^{2}\right), \xi^{1}, \xi^{2} \in D *$ is given by formula

$$
\xi(t)=\left(\xi_{1}(t), \xi_{2}(t)\right)=\left((1-t) \xi_{1}+t \xi_{1}^{2},(1-t) \xi_{2}^{1}+t \xi_{2}^{2}\right), 0 \leq t \leq 1
$$

Let us consider the preimage of this line segment in Reinhardt diagram. Since $\xi_{1}=\ln \left|z_{1}\right|$ and $\xi_{2}=\ln \left|z_{2}\right|$, it follows from (4) that

$$
\left\{\begin{array}{l}
\ln \left|z_{1}\right|=(1-t) \ln \left|z_{1}^{1}\right|+t \ln \left|z_{1}^{2}\right|, \quad 0 \leq t \leq 1 . \\
\ln \left|z_{2}\right|=(1-t) \ln \left|z_{2}^{1}\right|+t \ln \left|z_{2}^{2}\right|
\end{array}\right.
$$


Hence,

$$
\frac{\ln \left|z_{2}\right|-\ln \left|z_{2}^{1}\right|}{\ln \left|z_{1}\right|-\ln \left|z_{1}^{1}\right|}=\frac{\ln \left|z_{2}^{2}\right|-\ln \left|z_{2}^{1}\right|}{\ln \left|z_{1}^{2}\right|-\ln \left|z_{1}^{1}\right|}
$$

We denote by $k$ a quotient

$$
k=\frac{\ln \left|z_{2}^{2}\right|-\ln \left|z_{2}^{1}\right|}{\ln \left|z_{1}^{2}\right|-\ln \left|z_{1}^{1}\right|} .
$$

Without loss of generality we can assume that $\left|z_{1}^{1}\right| \leq\left|z_{1}^{2}\right|$. If $\left|z_{1}^{2}\right|=\left|z_{1}^{1}\right|$ (that is, $k= \pm \infty$ ) or $\left|z_{2}^{2}\right|=\left|z_{2}^{1}\right|$ (that is, $k=0$ ), then the curve (5) is line segment joining the points $\alpha\left(z^{1}\right)=\left(\left|z_{1}^{1}\right|,\left|z_{2}^{1}\right|\right)$ and $\alpha\left(z^{2}\right)=\left(\left|z_{2}^{1}\right|,\left|z_{2}^{2}\right|\right)$, which is parallel to vertical or horizontal axis, respectively. Since $G$ is convex, this line segment lies entirely in $G$. Therefore the correspondent line segment (4) is contained in $D^{*}$.

If the coordinates of the points $\alpha\left(z^{1}\right)$ and $\alpha\left(z^{2}\right)$ are different (that is, $k \neq 0, k \neq \pm \infty$ ), then equation (5) is transformed to a form

$$
\left|z_{2}\right|=\frac{\left|z_{2}^{1}\right|}{\left|z_{1}^{1}\right|^{k}}\left|z_{1}\right|^{k}=a\left|z_{1}\right|^{k}, a>0 .
$$

If $k=1$ then (6) is an equation of linear function passing through origin. Hence, a curve (5) is line segment connecting the points $\alpha\left(z^{1}\right)$ and $\alpha\left(z^{2}\right)$ and lying entirely in $G$. Therefore the correspondent line segment (4) is entirely contained in $D^{*}$.

To investigate the function (6) when $k \in R, k \neq 0, k \neq 1$, we calculate its derivatives 


$$
\left|z_{2}\right|^{\prime}=a k\left|z_{1}\right|^{k-1},\left|z_{2}\right|^{\prime \prime}=a k(k-1)\left|z_{1}\right|^{k-2}
$$

and consider separately two cases.

1) If $k<0$ then $\left|z_{2}\right|^{\prime}<0$ and $\left|z_{2}\right|^{\prime \prime}>0$, that is, the function (6) is strictly decreasing and concave upward (see Fig.3). Since $G$ is convex, the line segment joining the points $\alpha^{1}$ and $\alpha^{2}$ lies entirely in $G$. Since $D$ is complete Reinhardt domain, the set $G$ contains entire convex polygon with vertices $\alpha^{1}, \alpha^{2},\left(\left|z_{1}^{2}\right|, 0\right),(0,0)$ and $\left(0,\left|z_{2}^{1}\right|\right)$ (the shaded part of Fig.3). Therefore, the part of curve (6), which connects the points $\alpha^{1}$ and $\alpha^{2}$, lies in the set $G$ and, consequently, line segment (4) is contained in set $D^{*}$.

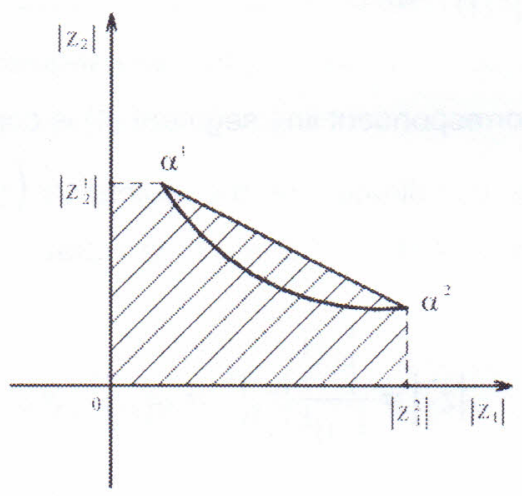

Fig. 3 - Curve (5) for $k<0$

2) If $k>0$ then $\left|z_{2}\right|>0$, that is, the function (6) is strictly increasing. It has downward concavity when $0<k<1$ and upward concavity when $k>1$. From $\left|z_{1}^{1}\right|<\left|z_{1}^{2}\right|$ and strict increasing of the function (6), it 
follows that $\left|z_{2}^{1}\right|<\left|z_{2}^{2}\right|$. Since $D$ is complete Reinhardt domain, together with the point $\alpha^{2}=\left(\left|z_{1}^{2}\right|,\left|z_{2}^{2}\right|\right)$ the set $G$ contains entire rectangle with vertices $(0,0),\left(0,\left|z_{2}^{2}\right|\right),\left(\left|z_{1}^{2}\right|, 0\right),\left(\left|z_{1}^{2}\right|,\left|z_{2}^{2}\right|\right)$. Since the point $\alpha^{1}=\left(\left|z_{1}^{1}\right|,\left|z_{2}^{1}\right|\right)$ belongs to indicated rectangle, the part of the curve (6), which joins the points $\alpha^{1}=\left(\left|z_{1}^{1}\right|,\left|z_{2}^{1}\right|\right)$ and, is contained in this rectangle too, and, consequently, in $G$ (see Fig.4). Therefore, line segment (4) lies in $D^{*}$.

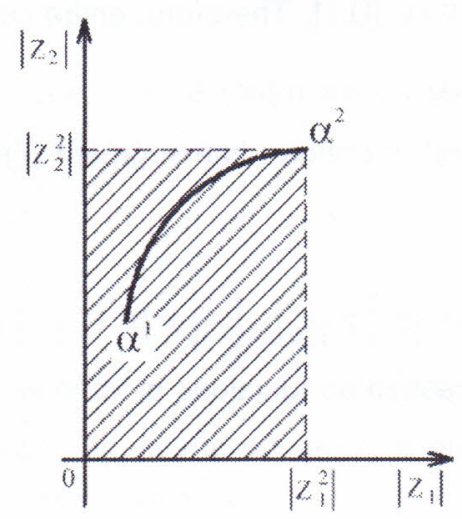

$(0<\mathrm{k}<1)$

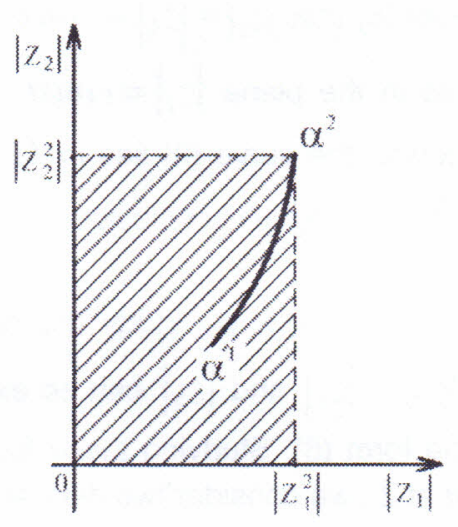

$(k>1)$

Fig. 4 - Curve (5) for $0<k<1$ and $k>1$

Thus, in the case of $n=2$ the theorem statement is proved.

2. Before to consider general case we study the case $n=3$.

Let $D^{*}$ be a logarithmic image of domain $D$. A line segment joining 
two arbitrary points $\xi^{1}=\left(\xi_{1}^{1}, \xi_{2}^{1}, \xi_{3}^{1}\right)$ and $\xi^{2}=\left(\xi_{1}^{2}, \xi_{2}^{2}, \xi_{3}^{2}\right)$, $\xi^{1}, \xi^{2} \in D^{*}$ is given by the formula

$$
\xi(t)=\left\{(1-t) \xi_{k}^{1}+t \xi_{k}^{2}\right\}_{k=1}^{3}, 0 \leq t \leq 1 .
$$

Without the loss of generality we set $\xi_{1}^{1} \leq \xi_{1}^{2}$. Let us consider preimages of the points $\xi^{1}, \xi^{2}$ and the interval (7) in the Reinhardt diagram:

$$
\begin{gathered}
\alpha^{1}=\alpha\left(z^{1}\right)=\left(\left|z_{1}^{1}\right|,\left|z_{2}^{1}\right|,\left|z_{3}^{1}\right|\right), \alpha^{2}=\alpha\left(z^{2}\right)=\left(\left|z_{1}^{2}\right|,\left|z_{2}^{2}\right|,\left|z_{3}^{2}\right|\right), \\
\ln \left|z_{k}\right|=(1-t) \ln \left|z_{k}^{1}\right|+t \ln \left|z_{k}^{2}\right|, k=1,2,3 ; 0 \leq t \leq 1 .
\end{gathered}
$$

Since $\xi_{1}^{1} \leq \xi_{1}^{2}$, it follows that $\left|z_{1}^{1}\right| \leq\left|z_{1}^{2}\right|$. If $\left|z_{1}^{1}\right|=\left|z_{1}^{2}\right|$, then it follows from (8) that $\left|z_{1}\right|=\left|z_{1}^{1}\right|=$ const, $\forall t \in[0 ; 1]$. Therefore, entire curve (8) lies in the plane $\left|z_{1}\right|=$ const, that is, we return to the case $n=2$. Hence, the curve (8) lies in $G$ and, therefore, line segment (7) lies in $D^{*}$. Analogous conclusions we obtain when $\left|z_{2}^{1}\right|=\left|z_{2}^{2}\right|$ or $\left|z_{3}^{1}\right|=\left|z_{3}^{2}\right|$.

Now we study the case $\left|z_{1}^{1}\right| \neq\left|z_{1}^{2}\right|,\left|z_{2}^{1}\right| \neq\left|z_{2}^{2}\right|,\left|z_{3}^{1}\right| \neq\left|z_{3}^{2}\right|$. By (8), $\left|z_{3}\right|$ and $\left|z_{2}\right|$ can be expressed as functions of variable $\left|z_{1}\right|$ in the form (6). Studying these functions in the same way as in the case $n=2$, we consider two different cases of behavior of curve (8).

1) $\left|z_{3}\right|=g\left(\left|z_{1}\right|\right)$ and $\left|z_{2}\right|=h\left(\left|z_{1}\right|\right)$ are increasing functions with respect to variable $\left|z_{1}\right|$. Then $\left|z_{1}^{2}\right|>\left|z_{1}^{1}\right|,\left|z_{2}^{2}\right|>\left|z_{2}^{1}\right|,\left|z_{3}^{2}\right|>\left|z_{3}^{1}\right|$. Since $D$ is complete Reinhardt domain and the point $\alpha^{2}=\left(\left|z_{1}^{2}\right|,\left|z_{2}^{2}\right|,\left|z_{3}^{2}\right|\right)$ belongs to set $G$, entire rectangular parallelepiped, whose opposite vertices are located in the point $\alpha^{2}$ and origin $O$ of coordinate 
system, is contained in set $G$, too. Then the point $\alpha^{1}$ belongs to this parallelepiped, that is, the curve ( 8 ) lies entirely in this parallelepiped and, consequently, in a set $G$. Hence, the line segment (7) is entirely contained in set $D^{*}$.

2) One of the above functions, for example, $\left|z_{2}\right|=h\left(\left|z_{1}\right|\right)$ is increasing and another $\quad\left|z_{3}\right|=g\left(\left|z_{1}\right|\right)$ is decreasing. Then $\left|z_{1}^{2}\right|>\left|z_{1}^{1}\right|,\left|z_{2}^{2}\right|>\left|z_{2}^{1}\right|,\left|z_{3}^{2}\right|<\left|z_{3}^{1}\right|$ and $\left|z_{3}\right|$ is decreasing function with respect to $\left|z_{2}\right|$, too. Since $G$ is a convex set, the line segment connecting the points $\alpha^{1}$ and $\alpha^{2}$ lies in $G$. We next consider a pyramid $A B C D E$ with vertices $A\left(\left|z_{1}^{1}\right|,\left|z_{2}^{1}\right|,\left|z_{3}^{1}\right|\right), B\left(\left|z_{1}^{2}\right|,\left|z_{2}^{1}\right|,\left|z_{3}^{2}\right|\right)$, $C\left(\left|z_{1}^{1}\right|,\left|z_{2}^{1}\right|,\left|z_{3}^{2}\right|\right), D\left(\left|z_{1}^{1}\right|,\left|z_{2}^{2}\right|,\left|z_{3}^{2}\right|\right)$ and $E\left(\left|z_{1}^{2}\right|,\left|z_{2}^{2}\right|,\left|z_{3}^{2}\right|\right)$ (see Fig.5).

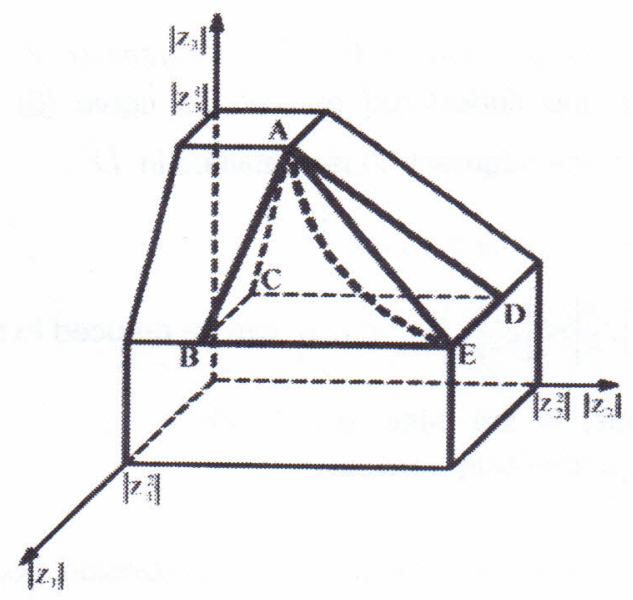

Fig. 5 - Curve (8), pyramid $A B C D E$ and convex shell. 
Since function $\left|z_{3}\right|=g\left(\left|z_{1}\right|\right)$ is strictly decreasing and concave upward, a projection of part of curve (8) between $\alpha^{1}$ and $\alpha^{2}$ (vertices $A$ and $E$ of pyramid) into plane $\left|z_{2}\right|=0$, lies below the projection of the line segment $A E$. Analogously, the function $\left|z_{3}\right|=\omega\left(\left|z_{2}\right|\right)$ is decreasing and concave upward and, therefore, a projection of the same part of the curve (8) into plane $\left|z_{1}\right|=0$ (that is, into plane $\left(\left|z_{2}\right|,\left|z_{3}\right|\right)$ ) lies below the projection of the line segment $A E$ into the same plane. Hence, entire part of the curve (8), lying between the points $\alpha^{1}$ and $\alpha^{2}$, is contained in pyramid $A B C D E$. Since $D$ is complete Reinhardt domain, together with any point of the line segment $A E$ it contains respective rectangular parallelepiped located in the positive octant $R_{+}^{3}$. Therefore, due to convexity of domain $D$, entire convex shell (convex polyhedron) spanned by the line segment $A E$ is contained in $G$. (Convex shell of an arbitrary set is the intersection of all convex sets which contain this given set. As a convex shell of the line segment $A E$ we consider a convex shell of the set of all rectangular parallelepipeds generated by the points of this line segment.)

Hence, pyramid $A B C D E$ is contained in the set $G$ and, consequently, the considered part of the curve (8) lies in $G$, too. Therefore, the line segment $(7)$ is contained in $D^{*}$.

The remaining cases $\left|z_{1}^{2}\right|>\left|z_{1}^{1}\right|,\left|z_{2}^{2}\right|<\left|z_{2}^{1}\right|,\left|z_{3}^{2}\right|>\left|z_{3}^{1}\right|$ or $\left|z_{1}^{2}\right|>\left|z_{1}^{1}\right|,\left|z_{2}^{2}\right|<\left|z_{2}^{1}\right|,\left|z_{3}^{2}\right|<\left|z_{3}^{1}\right|$ can be reduced to the case 2).

Thus, in the case $n=3$ any complete convex Reinhardt domain is logarithmically convex.

3) We now consider the case of arbitrary dimension. Let us suppose that theorem is true for $n-1$. As for $n=2$ and $n=3$ we consider 
Reinhardt diagram $G$ of domain $D \in C^{n}$ and logarithmic image $D^{*}$ of domain $D$ under logarithmic transformation (2).

Two arbitrary points $\xi^{1}=\left\{\xi_{k}^{1}\right\}_{k=1}^{n}$ and $\xi^{2}=\left\{\xi_{k}^{2}\right\}_{k=1}^{n}$ from set $D^{*}$ (that is, $\xi_{k}^{j}=\ln \left|z_{k}^{j}\right|, j=1,2 ; k=1, \ldots, n$ ) can be joined by line segment

$$
\xi(t)=\left\{(1-t) \xi_{k}^{1}+t \xi_{k}^{2}\right\}_{k=1}^{n}, 0 \leq t \leq 1
$$

whose preimage in Reinhardt diagram is

$$
\ln \left|z_{k}\right|=(1-t) \ln \left|z_{k}^{1}\right|+t \ln \left|z_{k}^{2}\right|, k=1, \ldots, n, 0 \leq t \leq 1 .
$$

The curve (10) connects the point $\alpha^{1}=\left\{\left|z_{k}^{1}\right|\right\}_{k=1}^{n}$ and $\alpha^{2}=\left\{\left|z_{k}^{2}\right|\right\}_{k=1}^{n}$, $\alpha^{1}, \alpha^{2} \in G$.

1) If the condition $\left|z_{k}^{1}\right|=\left|z_{k}^{2}\right|$ holds at least for one index $k$, $k=1, \ldots, n$, then entire curve (10) is contained in the intersection of set $G$ with plane $\left|z_{k}\right|=\left|z_{k}^{1}\right|=$ const, that is, in the space $R^{n-1}$.

Since any cross-section of convex set is convex set too, obtained intersection is Reinhardt diagram of complete convex Reinhardt domain in the space $C^{n-1}$. By induction supposition, the theorem is true in this case. Therefore the line segment (9) lies entirely in $D^{*}$.

2) In the case when all absolute values of the coordinates of one point are greater than correspondent absolute values of another one (for example, $\left.\quad\left|z_{k}^{2}\right|>\left|z_{k}^{1}\right|, \forall k=1, \ldots, n\right) \quad$ entire $\quad n$-dimensional parallelepiped with opposite vertices $\alpha^{2}$ and $O$ is contained in set $G$. In particular, the line segment joining the points $\alpha^{1}$ and $\alpha^{2}$ is contained in $G$. Entire curve (10) is contained in the parallelepiped with opposite vertices $\alpha^{1}$ and $\alpha^{2}$, that is, it is contained in $G$. Therefore, the line segment (9) lies entirely in $D^{*}$. 
3) If the coordinates of the points $\alpha^{1}$ and $\alpha^{2}$ satisfy inequalities with different signs, then, due to completeness of domain $D$, the set $G$ in the Reinhardt diagram contains entire rectangular parallelepiped with opposite vertices $\alpha^{1}$ and $O$. Analogously, the set $G$ contains entire rectangular parallelepiped with opposite vertices $\alpha^{2}$ and $O$. Moreover, since $G$ (like domain $D$ ) is convex set, $G$ contains a convex shell of the line segment between $\alpha^{1}$ and $\alpha^{2}$, that is, a convex shell spanned by respective rectangular parallelepipeds. Projection of the domain $D$ into any subspace $C^{n-1}$ defined by equation $z_{k}=0(\forall k=1, \ldots, n)$ is complete convex Reinhardt domain. Projections of the curve (10) into spaces $R^{n-1}\left(\left|z_{k}\right|=0, k=1, \ldots, n\right)$ are the curves of the same type lying in correspondent projection of the set $G$ by induction supposition. Since $G$ is diagram of complete Reinhardt domain, the curve (10) is contained in $G$. Hence, the line segment (9) lies entirely in $D^{*}$. Therefore, for any natural $n$, complete convex Reinhardt domain is logarithmically convex.

QED

Observation. Any convex Reinhardt domain is complete Reinhardt domain.

In fact, let $D$ be convex Reinhardt domain and $z^{0}$ be arbitrary point of this domain: $z^{0} \in D, z^{0}=\left(z_{1}^{0}, \ldots, z_{n}^{0}\right)$. Since $D$ is Reinhardt domain, it contains together with point $z^{0}$ all points in the form $z^{0}(\varphi)=\left(\left|z_{1}^{0}\right| e^{i \varphi_{1}}, \ldots,\left|z_{n}^{0}\right| e^{i \varphi_{n}}\right)$, where $\varphi_{k}$ are any arguments: $0 \leq \varphi_{k}<2 \pi, \forall k=1, \ldots, n$. In particular, the points $z^{1}=z^{0}(0)=\left(\left|z_{1}^{0}\right|, \ldots\left|z_{m}^{0}\right|\right) \quad$ and $z^{2}=z^{0}(\pi)=\left(\left|z_{1}^{0}\right| e^{i \pi}, \ldots,\left|z_{n}^{0}\right| e^{i \pi}\right)=\left(-\left|z_{1}^{0}\right|, \ldots,-\left|z_{n}^{0}\right|\right)$ belong to $D$. We consider the interval joining the points $z^{1}$ and $z^{2}$ : 


$$
z(t)=\left\{(1-t)\left|z_{k}^{0}\right|+t\left(-\left|z_{k}^{0}\right|\right)\right\}_{k=1}^{n}=\left\{(1-2 t)\left|z_{k}^{0}\right|\right\}_{k=1}^{n}, 0 \leq t \leq 1 .
$$

From convexity of $D$ and $z^{1}, z^{2} \in D$, it follows that $z(t) \in D, \forall t \in[0 ; 1]$. In particular, $z(t) \in D, \forall t \in[0 ; 1 / 2]$. For $0 \leq t \leq \frac{1}{2}$ the following inequalities hold $0 \leq\left|z_{k}(t)\right|=(1-2 t)\left|z_{k}^{0}\right| \leq\left|z_{k}^{0}\right|, \forall k=1, \ldots, n$. Since $D$ is Reinhardt domain, it contains together with any point $z(t)$ all points in the form $z=\left(\left|z_{1}(t)\right| e^{i \varphi_{1}}, \ldots,\left|z_{n}(t)\right| e^{i \varphi_{n}}\right)$, where $\varphi_{k}$ are any arguments: $0 \leq \varphi_{k}<2 \pi, \forall k=1, \ldots, n$. Hence, domain $D$ contains together with the point $z^{0}=\left\{z_{k}^{0}\right\}_{k=1}^{n}$ all points $z=\left\{z_{k}\right\}_{k=1}^{n}$ such that $\left|z_{k}\right| \leq\left|z_{k}^{0}\right|$, $\forall k=1, \ldots, n$, that is, domain $D$ is complete Reinhardt domain. QED

\section{Acknowledgements}

We are grateful to the science foundation FAPERGS for supporting this work with a grant 01/60053.9. 


\section{References}

[1] L. Bourchtein, A. Bourchtein, Some properties of Reinhardt and Hartogs Domains. UFSM, Ciencia e Natura, 2002, V.24, p.7-19.

[2] H. Cartan, Elementary Theory of Analytic Functions of One or Several Complex Variables. New York, Dover Pub., 1995, 226p.

[3] L. Hormander, An Introduction to Complex Analysis in Several Variables. Amsterdam, North-Holland, 1973, 211p.

[4] R. Narasimhan, Several Complex Variables. Chicago, University of Chicago Press, 1995, 174p.

[5] B.V. Shabat, Introduction to Complex Analysis. Part 2: Functions of Several Variables. Providence, American Mathematical Society, 1992, $381 \mathrm{p}$.

LUDMILA BOURCHTEIN

ANDREI BOURCHTEIN

Burstein@terra.com.br

Department of Mathematics, Pelotas State University

Campus Universitário da UFPel, Capão do Leão

Pelotas, RS, Brazil 